\title{
Subtle Oculomotor Difficulties and Their Relation to Motor Skill in Children with Autism Spectrum Disorder
}

\author{
Emma Sumner ${ }^{1}(\mathbb{D}) \cdot$ Samuel B. Hutton ${ }^{2}$ Elisabeth L. Hill ${ }^{3}$
}

Accepted: 24 October 2020 / Published online: 4 November 2020

(C) The Author(s) 2020

\begin{abstract}
Objectives Sensorimotor difficulties are often reported in autism spectrum disorders (ASD). Visual and motor skills are linked in that the processing of visual information can help in guiding motor movements. The present study investigated oculomotor skill and its relation to general motor skill in ASD by providing a comprehensive assessment of oculomotor control.

Methods Fifty children (25 ASD; 25 typically developing [TD]), aged 7-10 years, completed a motor assessment (comprising fine and gross motor tasks) and oculomotor battery (comprising fixation, smooth pursuit, prosaccade and antisaccade tasks).

Results No group differences were found for antisaccade errors, nor saccade latencies in prosaccade and antisaccade tasks, but increased saccade amplitude variability was observed in children with ASD, suggesting a reduced consistency in saccade accuracy. Children with ASD also demonstrated poorer fixation stability than their peers and spent less time in pursuit of a moving target. Motor skill was not correlated with saccade amplitude variability. However, regression analyses revealed that motor skill (and not diagnosis) accounted for variance in fixation performance and fast smooth pursuit.

Conclusions The findings highlight the importance of considering oculomotor paradigms to inform the functional impact of neuropathologies in ASD and also assessing the presentation of co-occurring difficulties to further our understanding of ASD. Avenues for future research are suggested.
\end{abstract}

Keywords Autism $\cdot$ Fixation $\cdot$ Motor skill $\cdot$ Oculomotor $\cdot$ Saccades $\cdot$ Smooth pursuit

Autism spectrum disorders (ASD) are characterized by difficulties with social interaction, together with restricted interests and repetitive behaviours (American Psychiatric Association [APA] 2013). Although not included in the diagnostic criteria, sensorimotor problems are also reported in a high proportion of individuals with ASD (e.g., Coll et al. 2020; Fournier et al. 2010; Mosconi and Sweeney 2015; Whyatt and Craig 2013), with some reporting motor impairments to be one of the earliest manifestations of this disorder (Landa et al. 2013; Sutera et al. 2007). Importantly, sensorimotor problems in this population have been shown to predict daily living skills (Travers et al. 2016), social function (Nebel et al. 2016) and language

Emma Sumner

e.sumner@ucl.ac.uk

1 Psychology and Human Development, UCL Institute of Education, London, UK

2 School of Psychology, University of Sussex, Brighton, UK

3 Department of Psychology, Goldsmiths, University of London, London, UK development (Bedford et al. 2015) and may even present an opportunity for therapeutic intervention (Chukoskie et al. 2013). As such, it is clear that a detailed understanding of sensorimotor deficits in ASD may provide important insights into its aetiology and treatment (Mosconi et al. 2015).

Given the nature of ASD, tasks requiring language or complex motor actions may hinder performance. One approach to overcome this, and at the same time to better understand sensorimotor function, is to use well-established oculomotor paradigms to examine the execution of oculomotor behaviour (fixations, saccades, and smooth pursuit). The oculomotor system has been described as a microcosm of the brain (Carpenter 2004), and research into oculomotor control has provided a window into sensorimotor and cognitive functioning in a range of neurological and neuropsychiatric disorders (Hutton and Ettinger 2006), as well as neurodevelopmental disorders (Karatekin 2007; Rommelse et al. 2008). Notably, some inconsistent reports of oculomotor performance can be found in the ASD literature (for a comprehensive review see Johnson et al. 2016). It is possible that ASD symptomology (i.e. the level of difficulty experienced or IQ level) may 
account for inconsistencies in the literature. For instance, in a prosaccade task, which requires participants to make a saccade (i.e. an eye movement) as quickly as possible to the location of a new peripheral target, Johnson et al. (2012) found that children identified as having high-functioning autism $(n=10)$ made more variable saccades to the target (which were hypometric at larger target amplitudes) in comparison to their peers. However, no group differences were observed on measures of saccade latency or peak velocity, leading the authors to suggest there are subtle oculomotor impairments in children with ASD. The finding of hypometric saccades and more variable primary saccade accuracies has also been reported in children with ASD aged 2-11 years (Kovarski et al. 2019), adolescents with autism (Rosenhall et al. 1988), and adults with ASD (Takarae et al. 2004a). Furthermore, the finding of no differences between adolescents and adults with and without ASD on measures of saccade latency or peak velocity is supported by earlier research (e.g., D'Cruz et al. 2009; Luna et al. 2007; Minshew et al. 1999; Takarae et al. 2004a). In contrast, however, reduced peak velocity in comparison to their peers has been reported in children and adults with ASD completing a prosaccade task (Schmitt et al. 2014), and greater latency for initiating saccades in a prosaccade task has been demonstrated by school-aged children with ASD when compared to their peers (Wilkes et al. 2015). High error rates are also consistently reported in adolescent and adult ASD samples when completing an antisaccade task (DiCriscio et al. 2016; Goldberg et al. 2002; Kelly et al. 2013; Kenet et al. 2012; Minshew et al. 1999; Mosconi et al. 2009) which taps into cognitive control processes (i.e. inhibitory control), as the individual must suppress a prosaccade towards a stimulus and instead execute a saccade in the opposite direction. This finding would suggest that adolescents and adults with ASD have difficulty with suppressing eye movement when a distraction is present and again hints towards oculomotor control difficulties.

Fewer studies have examined how adults with ASD perform on fixation tasks assessing gaze stability, but those that have report no differences to a control group on the frequency of saccades when fixating a central target and peripheral target (Frey et al. 2013; Nowinski et al. 2005; Takarae et al. 2007). Greater amplitudes of intrusive saccades, however, are noted in adults with ASD (Frey et al. 2013; Nowinski et al. 2005), which suggests subtle disturbances in the ability to maintain stable gaze (Shirama et al. 2016), a behaviour that is important for positioning visual information on the fovea and thus may affect how we process information in - and interact with - our environment. Exploration of gaze stability (fixation) in children with ASD would add to our understanding of top-down control and the integrity of brainstem-cerebellar networks in this population (Minshew et al. 1999).

The analysis of smooth pursuit eye movements has also revealed mixed results, although still few studies exist in this area. Using electrooculography (EOG), school-aged children with pervasive developmental disorder (PDD) have been reported as being comparable to a control group in velocity gain when tracking a horizontally moving target (Kemner et al. 2004). In contrast, on the same task but using infrared sensors mounted onto spectacles to record eye movements, young adults with ASD demonstrated lower pursuit velocity gain (i.e. poorer accuracy of open and closed loop gain) than their typically developing peers (Takarae et al. 2004b, 2007, 2008). Further, with the use of video-oculography goggles (VOG), Wilkes et al. (2015) found that school-aged children with ASD demonstrated significantly greater phase lag in vertical smooth pursuit trials than their peers, but the two groups were comparable on horizontal smooth pursuit trials. With very little known about how children with ASD perform in this respect (see Johnson et al. 2016), opportunities for early identification and support are missed.

Inconsistencies in previous research may partly be due to differences in the accuracy of methodologies used (EOG vs. video-based), differing and widespread age ranges, or the presence of co-occurring symptoms that have not been specifically tested and ruled out. Of particular interest here is the presence of co-occurring motor difficulties in ASD. Problems with motor timing and integration in ASD have gained considerable research and clinical interest over the past decade (Trevarthen and Delafield-Butt 2013). It is conceivable that oculomotor and motor skills are intertwined, as difficulties with tracking a moving object and control of eye movements would negatively impact on completion of motor tasks, such as catching/throwing a ball or navigating obstacles. Johnson et al. (2012) measured both general motor skill and oculomotor performance on saccade tasks in adolescent with ASD but did not explore the relationship between motor and oculomotor abilities. Research to date is yet to explicitly explore the relationship between oculomotor performance and wider motor skill in children with ASD.

The purpose of the present study, therefore, was twofold: (1) to provide a more comprehensive account of oculomotor function in children with ASD than currently exists in the literature, in comparison to typically developing (TD) children, investigating fixation stability, smooth pursuit, and pro-/antisaccades in a single study; and (2) to determine whether motor skill can predict oculomotor parameters found to be significantly different between children with and without ASD. Specifically, the oculomotor tasks (fixation stability, pursuit, pro-/antisaccades) were chosen given their widespread use in the adult literature (Johnson et al. 2016) and the lack of studies employing such tasks in child populations. For example, existing studies on antisaccade performance have largely focused on adults with only one considering adolescent behaviour (Kelly et al. 2013) and none with primaryaged children. Moreover, analysis of fixation stability has not been conducted on children with ASD. Further, by using a 
high-accuracy eye tracker, more sophisticated analyses quantifying the number of corrective (catch-up) and anticipatory (intrusive) saccades that are often made during pursuit can be computed. Together all four tasks provide a comprehensive overview of oculomotor control and knowledge of child performance may help to identify early characteristics of oculomotor difficulties which in turn will lead to earlier support.

\section{Methods}

\section{Participants}

Children (aged 7-10 years) formed two groups: 25 children with ASD (21 male) and 25 TD children (20 male). Background characteristics are presented in Table 1. All children had a Full Scale IQ (FSIQ) above 80 on the Wechsler Intelligence Scales for Children (WISC-IV; Wechsler 2003).

Children with ASD were recruited through a UK charitable foundation, the National Autistic Society, and primary schools in South London and had an existing and single diagnosis from relevant clinicians external to the study. In a background questionnaire, parents did not identify their child as having any additional diagnoses (developmental, visual impairment,

Table 1 Descriptive statistics for the background measures

\begin{tabular}{llll}
\hline & $\operatorname{TD}(n=25)$ & $\operatorname{ASD}(n=25)$ & $F(d f), p$ \\
\hline Age (years) & & & \\
Mean (SD) & $9.00(0.96)$ & $8.85(1.19)$ & $0.44(1.48) 0.51$ \\
Range & $7.74-10.69$ & $7.01-10.81$ & \\
ADOS score & & & \\
Mean (SD) & - & $8.57(1.51)$ & - \\
Range & & $7-10$ & \\
SCQ score & & & \\
Mean (SD) & $2.88(2.59)$ & $22.48(6.14) * *$ & $216.05(1.48)<0.001$ \\
Range & $0-9$ & $15-38$ & \\
FSIQ standard score & & \\
Mean (SD) & $109.04(10.69)$ & $100.92(15.04)^{*}$ & $4.84(1.48) 0.03$ \\
Range & $89-124$ & $80-134$ & \\
SDQ hyperactivity & & \\
Mean (SD) & $1.88(1.86)$ & $6.76(2.82)^{\mathrm{a} * *}$ & $52.30(1.48)<0.001$ \\
Range & $0-6$ & $0-10$ & \\
MABC-2 percentile & & \\
Mean (SD) & $65.40(21.55)$ & $34.72(33.08)^{* * *}$ & $15.09(1.48)<.001$ \\
Range & $25-98$ & $0.01-95$ & \\
\hline
\end{tabular}

ADOS Autism Diagnostic Observation Schedule. SCQ Social Communication Questionnaire. FSIQ Full Scale IQ, M 100, SD 15; $S D Q$ Strengths and Difficulties Questionnaire, $M A B C$-2 Movement Assessment Battery for Children, ${ }^{\mathrm{a}} 3$ substituted data points (parent responses used rather than teachers' because the latter did not return the questionnaire) medical). The ASD diagnosis was corroborated by a member of the research team who was trained to research reliability level to be able to administer the Autism Diagnostic Observation Schedule (ADOS-2; Lord et al. 2012). Children scored $\geq 7$ on Module 3 of the ADOS-2 (Lord et al. 2012). Three children in the ASD group did not complete the ADOS as part of the research because they had completed it recently as part of their diagnostic assessment. However, parents provided confirmation of the diagnostic statement and completed the Social Communication Questionnaire (SCQ; Rutter et al. 2003) whereby all children scored 15 or above to further confirm ASD symptomology. Although parents did not report cooccurring language impairment for any of the children with $\mathrm{ASD}$, it was recognized that previous research has found greater oculomotor difficulties in adolescents/adults recognized with ASD plus language delay than those with a single diagnosis of ASD (Kelly et al. 2013; Takarae et al. 2008). In the present sample, group differences (ASD vs TD) were noted for FSIQ (Table 1, $p=.03$ ); however, no group differences were found on the verbal comprehension index $(p=.34)$ of the WISC-IV, suggesting that the likelihood of significant language problems was reduced.

TD children were recruited through primary schools in South London and were matched in age to the ASD group. Parents of TD children identified no diagnoses of any kind and completed the SCQ to rule out ASD-related symptomatology (all scoring $<15$ cutoff).

To further characterize the groups, a measure of hyperactivity was taken using the scale from the Strengths and Difficulties Questionnaire, Teacher version (SDQ; Goodman 1997). Children with ASD scored significantly higher $(p<.001)$ on this scale than their peers despite not having an ADHD diagnosis. Motor competency was assessed using the Movement Assessment Battery for Children, second edition (MABC-2; Henderson et al. 2007), a standardized assessment of fine and gross motor skill with UK norms. The MABC-2 comprises age-appropriate tasks that assess manual dexterity, aiming and catching, and balance. Raw scores were converted to standard scores and percentiles. According to the test manual, a score $\leq 16$ th percentile is indicative of motor difficulties. TD children demonstrated age-appropriate motor skill $(\geq 25$ th percentile). However, 12 children in the ASD group were performed $\leq 16$ th percentile, with the remaining 13 children scoring $\geq 25$ th percentile. Of note, splitting the ASD group by the motor skill cutoff demonstrated that the two subgroups remained matched for age, FSIQ (including the verbal comprehension index), ADOS, SCQ, and SDQ performance (all $p>.05$ ), differing only in motor skill.

\section{Procedure}

This study was approved by Goldsmiths, University of London, ethics committee. Informed written parental consent 
was obtained, and children gave verbal assent once the project had been explained. Children were tested individually in a quiet room either at their school or at the research lab. Assessments were completed either on the same day (with sufficient breaks) or across two separate days. The session which focused on the oculomotor battery took between 20 and $30 \mathrm{~min}$. Teachers completed the SDQ, although 3 questionnaires related to children in the ASD group were not returned and in these cases parents' answers are used (see Table 1).

\section{Measures}

Oculomotor Battery Eye movements were recorded using the Eyelink 1000 eye tracker (SR-research; $1000 \mathrm{~Hz}$ sampling rate) operating in head-fixed mode (see Fig. 1). Children were seated at a viewing distance of approximately $80 \mathrm{~cm}$ and a combined forehead/chin rest was used to keep the head stable. Children completed a 5-point calibration at the beginning of the session, which was repeated as required. A drift correct procedure preceded each task and trial. The target was always a red circle (measuring $0.65^{\circ} \times 0.65^{\circ}$ visual angle, which equates to approximately $9 \times 9 \mathrm{~mm}$ on a $40 \times 30 \mathrm{~cm}$ screen at $80 \mathrm{~cm}$ distance) presented against a black background (1024 $\times 786$ screen resolution). Written and verbal instructions were provided for all children. Four tasks were presented in the following order, with breaks provided between tasks.

(i) In the visual fixation task, children were required to maintain their gaze on the target shown for $30 \mathrm{~s}$ in the centre of the screen, until it disappeared. (ii) Children completed two smooth pursuit tasks that lasted $20 \mathrm{~s}$ each. The target was presented at two speeds. They were instructed to follow the target (i.e. keep their eyes on the target) which had a horizontal sinusoidal motion starting in the centre and moving both left and right at $0.2 \mathrm{~Hz}$ (slow trial; target travelling $8.5^{\circ} / \mathrm{s}$ ) and then at $0.5 \mathrm{~Hz}$ (fast trial; target travelling $21.5^{\circ} / \mathrm{s}$ ). Finally, in the (iii) prosaccade (24 trials) and (iv) antisaccade (24 trials) tasks, the cue disappeared at the same time as the peripheral target

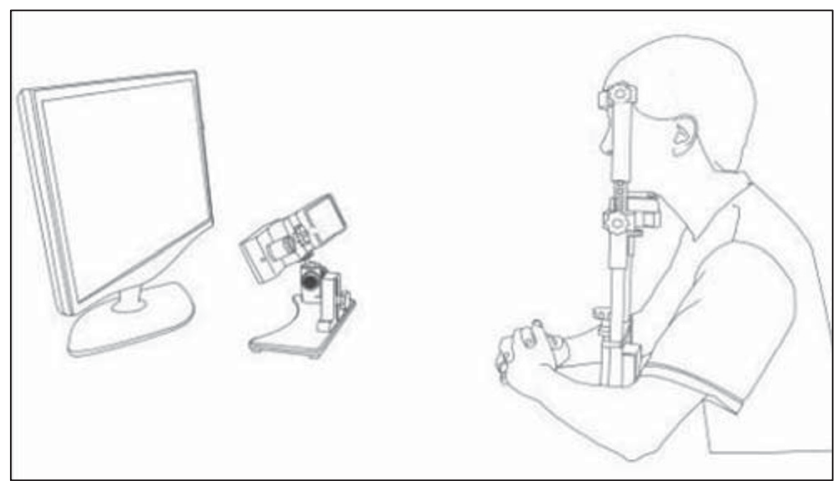

Fig. 1 An example of the eye tracking setup (recording device in a desktop mounted below the computer screen) appeared (see Fig. 2). The central target was displayed for $1000 \mathrm{~ms}$ before moving on the horizontal meridian $6.25^{\circ}$ to the left or right (direction was randomized). The target was displayed in the new location for $1000 \mathrm{~ms}$. For the prosaccade task, children were instructed to look at the central fixation point and then move their eyes as quickly as possible to where the target moved to. The presentation and timing of the target remained the same in the antisaccade task (no pseudo target was used), but this time children were instructed to ignore the target when it moved from the centre of the screen and instead to "quickly look in the opposite direction". The antisaccade instructions were fully explained to the child.

\section{Data Analyses}

The eye tracking experiment was implemented using Experiment Builder and analysed using Data Viewer (both SR Research software). Pursuit data were analysed using customized software written in LabView (software available on request). The details about the eye tracking analysis follow along with the statistical approach used.

Fixation Three measures were used to assess fixation stability: number of saccades made, average fixation duration, time on target (within $1^{\circ}$ visual angle). The EyeLink software makes online parsing decisions whereby saccades are classed as when the sample velocity exceeds $30^{\circ}$ per second.

Smooth Pursuit For both trials, four key metrics of smooth pursuit performance were used: number of pursuit segments, the sum of durations of these pursuit segments (i.e. time spent in pursuit), pursuit gain, and root-mean-square error (RMSE). Pursuit gain represents the average eye velocity divided by target velocity for all pursuit segments. RMSE was calculated as the square root of the average of the eye position subtracted from target position (in degrees of visual angle). Weighted averages, by the duration of pursuit segments, were used for the velocity gain and RMSE measures so that values from longer pursuit segments contribute more than values from shorter pursuit segments. The duration of each segment was determined using the online parsing decisions made by EyeLink (samples are identified as being a saccade if the sample velocity exceeds $30^{\circ}$ per second or acceleration exceeds $8000^{\circ}$ per second). All samples that were not classed as being part of a saccade or a blink (i.e. tracking loss) were considered a pursuit segment and based on these segments, gain, duration, and RMSE were calculated. In addition, tracking loss during smooth pursuit was calculated in case this contributed to performance and detailed analyses of the frequency of anticipatory saccades (classed as saccades that took the eye $>4^{\circ}$ ahead of the target) and corrective saccades (those that were $<4^{\circ}$ from the target-reducing position error) were considered. 
Fig. 2 Example of the pro- and antisaccade tasks
Prosaccade task

Start point: central fixation

New target: prosaccade movement

Antisaccade task

Start point: central fixation

Antisaccade movement

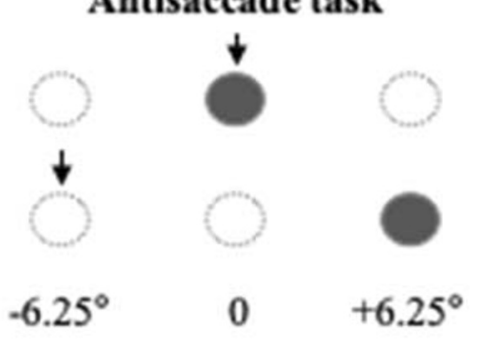

Shaded grey circles represent the location of the visual target shown to participants. The example shows only the target moving to the right but the direction in the trials was to both left and right at random. Dotted circles were not visible to participants. Arrows indicate the correct gaze position for both trials.
Prosaccade and Antisaccade The first step of analysis for these two tasks was to identify valid trials, which were only those that the participant was found to (1) fixate the central fixation point at target onset and (2) make a saccade $>80 \mathrm{~ms}$ to/away from the new target location (i.e. that could not be considered to be anticipatory). Only valid trials were then considered in the second step of analysis, which was to measure saccade latency $(\mathrm{ms})$ and percentage of direction errors. Accuracy was also measured in the prosaccade task by considering saccade amplitude (i.e. how close the eye lands to the target) as well as variable error of saccades (the standard deviation of saccade amplitude across trials). Variable error provides a measure of consistency of saccade endpoints (high values represent reduced movement consistency). Saccade amplitude is reported in degrees of visual angle (determined by the EyeLink) based on parameters for screen height, width, distance, and pixel resolution.

Statistical Approach Data were analysed using SPSS. Tests of normality were performed, and parametric tests were conducted unless otherwise indicated. The analysis process began with group comparisons (TD vs ASD) on each oculomotor measure. Given that the two groups differed in terms of the SDQ hyperactivity rating, as well as on the motor measure, the role of hyperactivity was also considered in later analysis. It was not appropriate to include the SDQ hyperactivity or MABC-2 motor measures as covariates in this first stage as the two groups differ statistically in this respect, thus violating assumptions for ANCOVA. Instead, correlational analyses were run on the oculomotor measures that yielded significant group differences to determine their relationship with hyperactivity and motor skill. Subsequently, multiple regression analysis was computed to determine the predictive value of age, motor skill, hyperactivity, and diagnosis on significant oculomotor measures.

\section{Results}

\section{Fixation}

ANOVAs were carried out on the first two fixation measures in Table 2. Children with ASD made significantly more saccades during the fixation task than their TD counterparts, $F(1,48)=6.18, p=.02, n^{2}{ }_{\mathrm{p}}=.11$, although the two groups performed similarly on average fixation duration, $F(1,48)=$ $3.59, p=.06, n_{\mathrm{p}}^{2}=.07$. A Mann-Whitney test revealed that

Table 2 Group comparisons of visual fixation stability

\begin{tabular}{lll}
\hline & $\begin{array}{l}\text { TD }(n=25) \\
\text { Means (SD) }\end{array}$ & $\begin{array}{l}\text { ASD ( } n=25) \\
\text { Means (SD) }\end{array}$ \\
\hline Number of saccades & $22.52(12.02)$ & $33.60(18.76)^{*}$ \\
Average fixation duration (s) & $1.77(1.93)$ & $1.07(0.77)$ \\
Time on target (\%) & $86 \%$ & $70 \% *$ \\
\hline
\end{tabular}

$\mathrm{s}=$ seconds. $* p<.05$ 
children with ASD spent significantly less time on target than their peers, $U=208.00, z=-2.03, p=.02$.

\section{Smooth Pursuit}

A 4 (pursuit measures: number of segments, duration, gain, RMSE) $\times 2$ (speed: slow vs fast) mixed ANOVA was performed with group as the between-subjects factor (mean scores in Table 3). There was a main effect of the pursuit measures, $F(2,45)=317.37, p<.001, n^{2}=.93$, and a main effect of the speed of the pursuit trial, $F(1,46)=48.60$, $p<.001, n_{p}^{2}=.51$, revealing better performance on the slower trial $(p<.001)$. There was a main effect of group, $F(1,46)=$ 17.67, $p<.001, n_{p}^{2}=.28$, and a significant interaction between the pursuit measures and group, $F(2,45)=11.13$, $p<.001, n_{p}^{2}=.33$, but not for speed and group $(p=.96)$. Bonferroni-corrected ANOVAs $(p=.006)$ revealed that children with ASD spent significantly less time in pursuit (i.e. pursuit duration) than TD children in the slow $(p<.001)$ and fast $(p=.003)$ trials, and the ASD group demonstrated significantly lower pursuit gain on the fast trial $(p<.001)$. For all other measures, the groups were comparable ( $p$ s $>.03$ ).

Tracking loss during smooth pursuit was similar for both groups, TD slow trial $M=1.94 \mathrm{~s}$, fast trial $M=.84 \mathrm{~s}$; ASD slow trial $M=1.50 \mathrm{~s}$, fast trial $M=.95 \mathrm{~s}$, and thus poorer performance in the ASD group could not be attributed to spending less time looking at the computer screen. Moreover, Fig. 3 highlights that children with ASD made more anticipatory saccades (i.e. saccades $>4^{\circ}$ ahead of the target) than TD children, in both tasks. The percentage of type of saccades was calculated in relation to the overall number of saccades made across both trials in Fig. 3 .

Table 3 Group comparisons of smooth pursuit performance

\begin{tabular}{lcc}
\hline & TD $(n=25)$ & ASD $(n=25)$ \\
Means (SD) & Means (SD) \\
\hline Slow trial & & \\
Number of segments & $20.17(5.61)$ & $19.64(6.08)$ \\
Pursuit duration (s) & $15.29(2.13)$ & $11.40(3.74)^{*}$ \\
Average gain $\dagger$ & $1.02(0.07)$ & $0.99(0.08)$ \\
Average RMSE $\dagger$ & $0.71(0.17)$ & $0.74(0.28)$ \\
Fast trial & & $20.92(13.70)$ \\
Number of segments & $27.96(6.75)$ & $7.63(4.98)^{*}$ \\
Pursuit duration (s) & $11.45(3.13)$ & $0.86(0.09)^{*}$ \\
Average gain $\dagger$ & $0.98(0.08)$ & $1.11(0.19)$ \\
Average RMSE $\dagger$ & $1.20(0.12)$ & \\
\hline
\end{tabular}

$\uparrow$ Averages are weighted so that larger values contribute more than smaller values. $\mathrm{s}=$ seconds. Larger gain values $=$ better pursuit; lower RMSE $=$ better pursuit. $* p<.05$

\section{Prosaccade and Antisaccade}

Out of 24 trials in both tasks, the mean (SD) number of valid trials in the prosaccade condition were: TD, 21.00 (1.91) and ASD, 19.45 (3.37); and for the antisaccade condition: TD, 19.08 (3.91) and ASD, 17.36 (5.79). Analysis of prosaccade amplitude revealed no group differences, $F(1,46)=.96$, $p=.33, n^{2}{ }_{\mathrm{p}}=.02$ (TD: $M=6.81^{\circ}, S D=.53$; ASD, $M=6.89^{\circ}$, $S D=.77)$. However, variable error was significantly higher in children with ASD, indicating reduced consistency in saccades made to the target, $F(1,46)=9.03, p=.004, n^{2}=.17$ (TD: $M=.78, S D=.19$; ASD: $M=1.49, S D=1.18$ ).

Latency and error rate results for the prosaccade and antisaccade tasks are shown in Fig. 4. A 2 (group: TD vs ASD) $\times 2$ (task: prosaccade vs antisaccade) ANOVA revealed a significant effect of task on latency scores, $F(1,44)=22.64$, $p<.001, n_{\mathrm{p}}^{2}=.34$, with faster latencies in the prosaccade task $(p<.001)$. There was a non-significant effect of group, $F(1,44)=.04, p=.84, n^{2}{ }_{p}=.01$, and no interaction was found, $F(1,44)=.01, p=.94, n_{\mathrm{p}}^{2}=.01$. Direction errors were not common in the prosaccade task (1 TD child made 1 error; 1 ASD child made 5 errors); therefore statistical analyses of group differences focused on the antisaccade task. No group differences were found for antisaccade direction errors, $F(1,47)=1.95, p=.17, n_{p}^{2}=.04$. Overall, ASD and TD children performed similarly on both tasks.

\section{Associations Between Motor Skill and Hyperactivity with Oculomotor Performance}

The oculomotor measures that revealed significant group differences above were entered into a correlation analysis alongside motor ability and the measure of hyperactivity (SDQ) (Table 4: Bonferroni critical alpha: $p<.005$ ). The two groups were combined for this analysis, given the smaller sample size if split. Saccade count and the time on target in the fixation task were highly correlated; therefore, the decision was taken to use the latter measure as it gives a better indication of adhering to the nature of the task.

Motor ability positively correlated with the time on target in the fixation task, as well as with the duration of pursuit and gain in the fast trial of the smooth pursuit task (moderate correlations were observed, $r=.41-.43$ ). No other correlations were significant for motor skill. In contrast, hyperactivity was only found to negatively correlate with time on target in the fixation task and the duration of pursuit in the slow trial of the smooth pursuit task.

\section{Regressions}

Based on the significant correlations, three oculomotor parameters (fixation time on task, duration of pursuit in slow and fast speeded trials) were considered as outcome measures in 
Fig. 3 Proportion of anticipatory and corrective saccades made

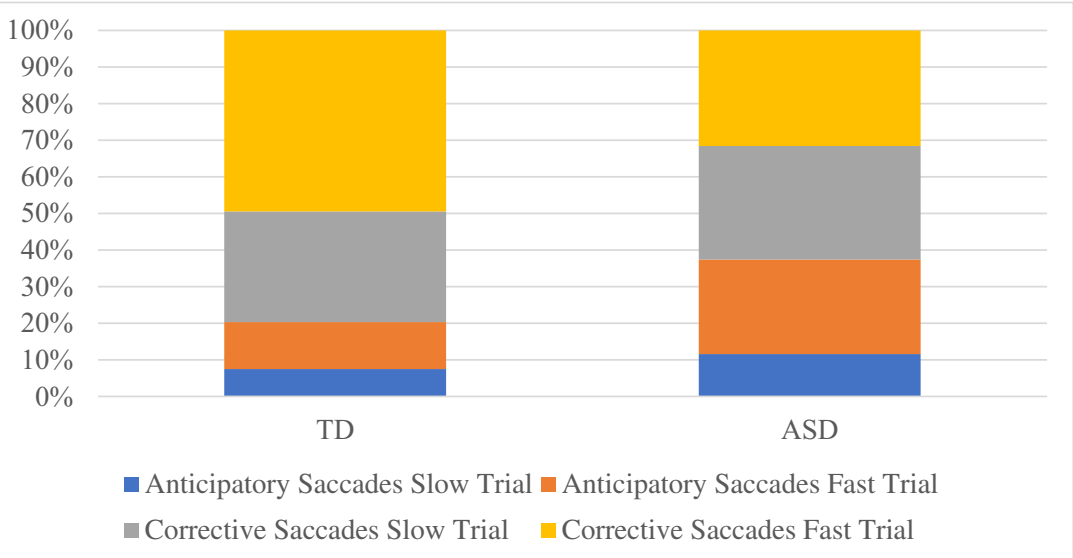

regression analyses. Pursuit gain was not considered to reduce the number of statistical tests conducted and it was felt that this measure was being covered by the duration.

Using stepwise regression age, motor ability (MABC-2 overall composite score) and hyperactivity (SDQ subscale) measures were always entered at the first step, with group entered at the final step to examine whether diagnosis predicted additional variance after accounting for the previous measures. Given that three regressions were conducted, the significance of each final model was assessed against a Bonferronicorrected critical value of $p=0.016(0.05 / 3)$. A summary of the final step in each model is presented in Table 5. Of note,
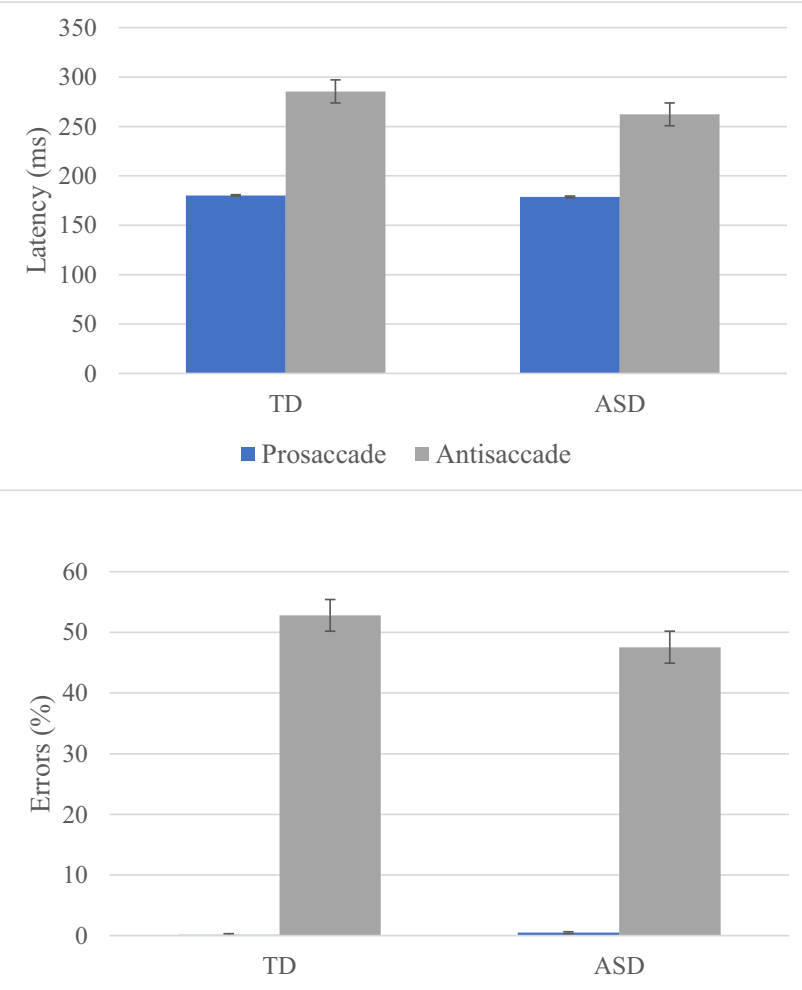

Fig. 4 Latency (ms) and error rates for the prosaccade/antisaccade tasks age was removed from the output of the stepwise analysis in each case and therefore is not reported as a predictor variable.

The results demonstrated a significant model for fixation time on task, $F(3,46)=5.20, p=.004$, with the measures predicting $25 \%$ of the variance overall, as well as for the measure of duration in pursuit in the fast pursuit trial, $F(3,46)=4.97, p=.005$, predicting $25 \%$ of the variance overall. In both models, only motor ability was a significant predictor. Adding group to the model did not represent a better model fit overall (change in $R^{2}$ at step 2, $p>.05$ ) indicating group differences had been accounted for in the previous step of the model (i.e. by motor skill). In contrast, a significant model emerged for the smooth pursuit slow duration, $F(3,46)=6.96, p=.001$, predicting $32 \%$ of the variance, but hyperactivity was the significant predictor at the first step (not motor skill) and adding the group variable at step 2 did represent a better fit of the model overall $\left(R^{2}\right.$ change, $\left.p<.05\right)$.

\section{Discussion}

The present study aimed to provide a detailed account of oculomotor control in children with ASD in comparison to TD children, employing a considerably tighter age range than existing studies. A second aim was to examine the influence of motor skill in predicting oculomotor parameters which were found to be significantly different between children with and without ASD. Following the initial group analysis, where hyperactivity was found to be higher in children with ASD in the present sample, the decision was also taken to consider the role of hyperactivity in predicting oculomotor parameters although, of note, children with ASD did not have an additional diagnosis of attention difficulties (i.e. ADHD).

The findings suggest that children with ASD present with subtle oculomotor difficulties. Children with ASD were comparable to their peers on horizontal pursuit gain, RMSE, and the number of pursuit segments in the slower of the two pursuit trials, as well as RMSE and the number of pursuit 
Table 4 Correlations between motor ability, hyperactivity, and selected oculomotor measures

\begin{tabular}{lll}
\hline & MABC-2\%ile & SDQ hyperactivity \\
\hline Fixation: time on target & $r=0.431, p=0.002^{*}$ & $r=-0.425, p=0.002^{*}$ \\
Smooth pursuit: slow duration & $r=0.380, p=0.008$ & $r=-0.479, p=0.001^{*}$ \\
Smooth pursuit: fast duration & $r=0.439, p=0.002^{*}$ & $r=-0.286, p=0.048$ \\
Smooth pursuit: fast gain & $r=0.415, p=0.003^{*}$ & $r=-0.393, p=0.006$ \\
Prosaccade: variable error & $r=-0.129, p=0.393$ & $r=0.301, p=0.042$ \\
\hline
\end{tabular}

$* p<0.005$

segments in the faster trial. These findings support existing studies of school-aged children that measured gain using different methodologies (EOG: Kemner et al. 2004; VOG: Wilkes et al. 2015). However, in our study, group differences were observed for the duration of time spent in pursuit in both speeded trials, as well as in pursuit gain on the faster trial. Comparisons across different target speeds have not been made in previous studies. Proficiency in pursuit gain when tracking a slow target suggests that the kinematics of this eye movement are mature at least to some extent, but that this system is easily compromised when demands are higher. Less time spent in pursuit across both speeds and more anticipatory saccades (overshooting the target) observed in children with ASD further suggest a difficulty with maintaining attentional focus on the target, a finding that has been found previously in children with motor difficulties (i.e. developmental coordination disorder: Sumner et al. 2018). Children with ASD were also shown to have poorer fixation stability than their peers in the fixation task, making more saccades and thus spending less time on the target. This finding contrasts with findings from ASD adult populations (Frey et al. 2013; Nowinski et al. 2005; Takarae et al. 2007) but adds to the profile of children with ASD potentially struggling with attending to one target for a period of time.

That said, children with ASD performed similarly to their peers on the measures of saccade accuracy, latency, and error rates from the prosaccade and antisaccade tasks. These findings suggest that children with ASD did not have difficulty with initiating (latency) and planning saccades towards a target, supporting existing adolescent and adult data (D'Cruz et al. 2009; Goldberg et al. 2002; Johnson et al. 2016; Takarae et al. 2004a, b). However, increased saccade amplitude variability in the ASD group supports existing findings (Johnson et al. 2012) and highlights reduced eye movement consistency. Johnson et al. (2016) argued that greater variability of saccades demonstrates difficulties with recalibrating saccade inaccuracies across trials and is suggestive of disruption between the cerebellar vermis-fastigial nuclei pathway and the brainstem premotor nuclei. This could be considered in future research. In addition, the conflicting antisaccade error rate results in relation to existing studies that found more directional errors made in adolescents (Goldberg et al. 2002) and adults (DiCriscio et al. 2016) with ASD may be due to the present study systematically excluding children with co-occurring diagnoses (although teacher ratings of hyperactivity scored higher as a group), which others did not, and as a result we find no differences between TD and ASD groups on suppressing reflexive saccades to a distracting target. This is interesting given that in the fixation and smooth pursuit trials, our results suggest difficulty with attention in the ASD group (noting more saccades in the absence of a distractor) but here no problems with inhibition (in the presence of a distractor) are found.

Table 5 Summary of regression analyses predicting performance on three oculomotor measures

\begin{tabular}{|c|c|c|c|c|}
\hline \multirow[b]{2}{*}{ Oculomotor measure } & \multirow[b]{2}{*}{ Final model Adjusted $R^{2}$} & \multicolumn{2}{|l|}{ Step 1} & \multirow{2}{*}{$\begin{array}{l}\text { Step } 2 \\
\text { Group }\end{array}$} \\
\hline & & Motor ability & Hyperactivity & \\
\hline Fixation: time on target & $\begin{array}{l}0.25 * \\
p=0.004\end{array}$ & $\begin{array}{l}B=0.301, t=2.13 \\
p=0.04 *\end{array}$ & $\begin{array}{l}B=-0.291, t=-1.58 \\
p=0.12\end{array}$ & $\begin{array}{l}B=0.007, t=.03 \\
p=0.97\end{array}$ \\
\hline Smooth pursuit slow duration & $\begin{array}{l}0.32 * * \\
p<0.001\end{array}$ & $\begin{array}{l}B=0.13, t=.90 \\
p=0.37\end{array}$ & $\begin{array}{l}B=-0.386, t=-2.69 \\
p=0.01\end{array}$ & $\begin{array}{l}B=-0.36, t=-1.95 \\
p=0.05^{*}\end{array}$ \\
\hline Smooth pursuit fast duration & $\begin{array}{l}0.25^{*} \\
p=0.005\end{array}$ & $\begin{array}{l}B=0.389, t=.261 \\
p=0.01 *\end{array}$ & $\begin{array}{l}B=-0.110, t=-0.738 \\
p=0.60\end{array}$ & $\begin{array}{l}B=-0.336, t=-1.72 \\
p=0.09\end{array}$ \\
\hline
\end{tabular}

Age was excluded in the stepwise approach and therefore is not reported above. Standardized coefficients and $t$ values are provided for each predictor in step 2 , above significance values. $* p<.05, * * p<.001$ 
Of note, approximately half of the ASD sample (48\%) scored below cutoff for a motor difficulty. This value is slightly lower than other studies using the same assessment (79\% in Green et al. 2009; 65\% in Hilton et al. 2007). Moreover, using the SDQ, teachers were found to rate more hyperactive characteristics in the ASD sample (32\% scoring in the high range) than the comparison group. ASD has been shown to frequently co-occur with ADHD (Grzadzinski et al. 2011) but parents of the children in the present study did not report any additional diagnosis of ADHD. Interestingly, motor skill and hyperactivity did not relate to more variable saccades in the prosaccade task, suggesting that this may be characteristic of ASD symptomology. Significant correlations between motor skill, hyperactivity, and the oculomotor measures revealed that, as a whole, children with better motor skills spent more time on target (fixation) and in pursuit of the faster moving stimuli (smooth pursuit), while children with more hyperactive characteristics spent less time focusing on the target (fixation) and in pursuit of the slow-moving target (smooth pursuit). Regression analyses further revealed that, where there were group differences in the oculomotor parameters, motor skill was a significant predictor of fixation time on target and duration of pursuit in the fast trial. Adding group to the model did not result in a better overall fit, suggesting that group differences had been accounted for by motor skill and not further by diagnosis. In contrast, motor skill did not predict performance in the measure of duration of pursuit in the slower trial and, instead, hyperactivity related to performance here with group difference still existing after taking this into account. The findings stress the importance of exploring the presentation of co-occurring difficulties to better our understanding of autism spectrum disorders and to inform intervention appropriately. Similarly, oculomotor paradigms may be used to inform the functional impact of neuropathologies in ASD and considering both oculomotor and motor skill takes a more holistic approach to understanding ASD.

The findings demonstrated the role of wider motor skills and hyperactivity in oculomotor function in children with autism. They may suggest that the same networks that govern general motor movements overlap with oculomotor networks. Mechanisms behind motor control and eye movement control are widely distributed neuroanatomically, but both implicate the cerebellum - indicating a degree of shared neural networks (Diamond 2000; Mosconi et al. 2013; Takarae et al. 2004a, b). Thus, saccade and pursuit parameters can inform our understanding of the integrity of cerebellar networks in ASD. The role of the cerebellum with respect to oculomotor impairments in autism is questioned to some extent by the present findings, as proficient saccade dynamics in the prosaccade task and relative pursuit gain performance in the slow speed trial for children are not consistent with suggestions of cerebellar dysfunction. However, problems with higher pursuit velocity and intrusive saccades during fixation suggest atypicalities in cerebellar-brainstem circuitry and posterior lobules VI and VII of the vermis (as identified by others: Mosconi et al. 2013; Schmitt et al. 2014; Shirama et al. 2016). Indeed, the brainstem structure has been found to be larger in sample of early primary-aged children with ASD than their same age peers, which leads the authors to suggest that sensory and motor integration in children with ASD may be disrupted at the level of the brainstem (Bosco et al. 2019). Our present findings add to our understanding of the neuroanatomical basis in childhood ASD but could be further extended by using the oculomotor tasks in conjunction with MRI analyses that describe functional brain organization in samples with and without motor difficulties. This in turn would shed light on the neuropathology that might subserve oculomotor and broader motor control networks.

\section{Limitations and Future Research Directions}

A limitation of the present work is that it cannot provide a causal account of the link between oculomotor control and motor skill. Future research would benefit from considering whether early deficits in oculomotor control not only influence joint attention and social interaction (Brenner et al. 2007) but also motor control in some way for children with ASD. Gowen and Hamilton (2013) argue that the motor difficulties experienced by children with ASD result from inefficient perceptual input and poor sensorimotor integration. Accurate eye movements aid integration of vision and sensorimotor skills that are critical when planning limb movements. If children have difficulty maintaining sustained engagement on a stimulus, this would surely impact on acquisition of motor skill and precision. Alternatively, it could be argued that poor motor development neurologically impacts other aspects of development and the findings reported here may be suggestive of neural disturbance that links to motor problems, rather than oculomotor problems being a contributory factor per se. Future research should examine these skills longitudinally and seek to identify functional correlates of eye movement measures.

It is also recognized that the tasks in the present study were controlled and did not capture children's oculomotor skills in a naturalistic, free-viewing situation. Interestingly, a recent study which examined oculomotor function while individuals with ASD (aged 6-30) viewed short dynamic video clips revealed that those with ASD had increased variation in saccade accuracy and decreased saccade amplitude in comparison to their peers (Bast et al. 2020). Similar to the present study, the authors suggest that individuals with ASD lack precise coordination of the saccadic system and extend this by showing how this results in reduced visual exploration of a scene. It would be of interest to extend these results to testing the smooth pursuit system in a more naturalistic context and to test and how this relates directly to motor performance (e.g., 
the tracking of a moving ball before catching). Finally we must acknowledge that the present ASD sample had average IQ skills and no co-occurring language difficulties which may limit the generalizability of the findings to a broader ASD population. It would be useful to understand whether further subgroups exist within this population in relation to oculomotor control. However, this study does emphasize the importance of considering the presentation of co-occurring motor difficulties to further our understanding of ASD. Understanding oculomotor and motor control in ASD will direct intervention in a clinically meaningful way.

Acknowledgements Special thanks go to the participating children, parents, and schools.

Authors' Contributions ES designed and executed the study, conducted the data analysis, and wrote the paper. SH collaborated with the design (particularly the eye tracking methods) and writing of the paper. EH was involved in the conceptualization of the study and collaborated with the writing of the paper. All authors approved the final version of the manuscript for submission.

Funding This study was funded by The Leverhulme Trust (RPG-2012742).

\section{Compliance with Ethical Standards}

All procedures performed were in accordance with the ethical standards of the institutional and/or national research committee and with the 1964 Helsinki declaration and its later amendments or comparable ethical standards. Ethical approval was obtained from Goldsmiths, University of London, research ethics committee. Informed consent was obtained from all individual participants included in the study.

Conflict of Interest On behalf of all authors, the corresponding author states that there is no conflict of interest.

Open Access This article is licensed under a Creative Commons Attribution 4.0 International License, which permits use, sharing, adaptation, distribution and reproduction in any medium or format, as long as you give appropriate credit to the original author(s) and the source, provide a link to the Creative Commons licence, and indicate if changes were made. The images or other third party material in this article are included in the article's Creative Commons licence, unless indicated otherwise in a credit line to the material. If material is not included in the article's Creative Commons licence and your intended use is not permitted by statutory regulation or exceeds the permitted use, you will need to obtain permission directly from the copyright holder. To view a copy of this licence, visit http://creativecommons.org/licenses/by/4.0/.

\section{References}

American Psychiatric Association. (2013). Diagnostic and statistical manual of mental disorders (DSM, 5th). Arlington: American Psychiatric Association.

Bast, N., Mason, L., Freitag, C. M., Smith, T., Portugal, A. M., Poustka, L., Banaschewski, T., Johnson, M., \& The EU-AIMS LEAP Group. (2020). Saccade dysmetria indicates attenuated visual exploration in autism spectrum disorder. Journal of Child Psychology and Psychiatry. Advance of Print. https://doi.org/10.1111/jcpp.13267.

Bedford, R., Pickles, A., \& Lord, C. (2015). Early gross motor skills predict the subsequent development of language in children with autism spectrum disorder. Autism Research, 9, 991-1001. https:// doi.org/10.1002/aur.1587.

Bosco, P., Giuliano, A., Delafield-Butt, J., Muratori, F., Calderoni, S., \& Retico, A. (2019). Brainstem enlargement in preschool children with autism: Results from an intermethod agreement study of segmentation algorithms. Human Brain Mapping, 40, 7-19. https://doi.org/ $10.1002 / \mathrm{hbm} .24351$

Brenner, L. A., Turner, K. C., \& Müller, R.-A. (2007). Eye movement and visual search: Are there elementary abnormalities in autism? Journal of Autism and Developmental Disorders, 37, 1289-1309. https://doi.org/10.1007/s10803-006-0277-9.

Carpenter, R. (2004). The saccadic system: A neurological microcosm. $A C N R, 4,6-7$.

Chukoskie, L., Townsend, J., \& Westerfield, M. (2013). Motor skill in autism spectrum disorders: A subcortical view. International Review of Neurobiology, 113, 207-249. https://doi.org/10.1016/B978-0-12418700-9.00007-1.

Coll, S.-M., Foster, N. E. V., Meilleur, A., Brambati, S. M., \& Hyde, K. L. (2020). Sensorimotor skills in autism spectrum disorder: A metaanalysis. Research in Autism Spectrum Disorders, 76, 1010570. https://doi.org/10.1016/j.rasd.2020.101570.

D’Cruz, A. M., Mosconi, M. W., Steele, S., Rubin, L. H., Luna, B., Minshew, N., \& Sweeney, J. A. (2009). Lateralized response timing deficits in autism. Biological Psychiatry, 66, 393-397. https://doi. org/10.1016/j.biopsych.2009.01.008.

Diamond, A. (2000). Close interrelation of motor development and cognitive development and of the cerebellum and prefrontal cortex. Child Development, 71, 44-56. https://doi.org/10.1111/1467-8624. 00117.

DiCriscio, A. S., Miller, S. J., Hanna, E. K., Kovac, M., Turner-Brown, L., Sasson, N. J., Sapyta, J., Troiani, V., \& Dichter, G. S. (2016). Brief report: Cognitive control of social and nonsocial visual attention in autism. Journal of Autism and Developmental Disorders, 46, 2797-2805. https://doi.org/10.1007/s10803-016-2804-7.

Fournier, K. A., Hass, C. J., Naik, S. K., Lodha, N., \& Cauraugh, J. H. (2010). Motor coordination in autism spectrum disorders: A synthesis and meta-analysis. Journal of Autism and Developmental Disorders, 40, 1227-1240. https://doi.org/10.1007/s10803-0100981-3.

Frey, H. P., Molholm, S., Lalor, E., Russo, N. N., \& Foxe, J. J. (2013). Atypical cortical representation of peripheral visual space in children with an autism spectrum disorder. European Journal of Neuroscience, 38, 2125-2138. https://doi.org/10.1111/ejn.12243.

Goldberg, M. C., Lasker, A. G., Zee, D. S., Garth, E., Tien, A., \& Landa, R. J. (2002). Deficits in the initiation of eye movements in the absence of a visual target in adolescents with high functioning autism. Neuropsychologia, 40, 2039-2049. https://doi.org/10.1016/ s0028-3932(02)00059-3.

Goodman, R. (1997). The extended version of the strengths and difficulties questionnaire as a guide to child psychiatric caseness and consequent burden. Journal of Child Psychology and Psychiatry, 40, 791-801.

Gowen, E., \& Hamilton, A. (2013). Motor abilities in autism: A review using a computational context. Journal of Autism and Developmental Disorders, 43, 323-344. https://doi.org/10.1007/ s10803-012-1574-0.

Green, D., Charman, T., Pickles, A., Chandler, S., Loucas, T., Simonoff, E., \& Baird, G. (2009). Impairment in movement skills of children with autistic spectrum disorders. Developmental Medicine and Child Neurology, 51, 311-316. https://doi.org/10.1111/j.14698749.2008.03242.x. 
Grzadzinski, R., Di Martino, A., Brady, E., Mairena, M. A., O’Neale, M., Petkova, E., Lord, C., \& Castellanos, F. X. (2011). Examining autistic traits in children with ADHD: Does the autism spectrum extend to ADHD. Journal of Autism and Developmental Disorders, 41, 1178-1191. https://doi.org/10.1007/s10803-010-1135-3.

Henderson, S. E., Sugden, D. A., \& Barnett, A. L. (2007). Movement assessment battery for children (2nd ed.). London: The Psychological Corporation.

Hilton, C., Wente, L., LaVesser, P., Ito, M., Reed, C., \& Herzberg, G. (2007). Relationship between motor skill impairment and severity in children with Asperger syndrome. Research in Autism Spectrum Disorders, 1, 339-349. https://doi.org/10.1016/j.rasd.2006.12.003.

Hutton, S. B., \& Ettinger, U. (2006). The antisaccade task as a research tool in psychopathology: A critical review. Psychophysiology, 43, 302-313. https://doi.org/10.1111/j.1469-8986.2006.00403.x.

Johnson, B. P., Rinehart, N. J., Papadopoulos, N., Tonge, B., Millist, L., White, O., \& Fielding, J. (2012). A closer look at visually guided saccades in autism and Asperger's disorder. Frontiers in Integrative Neuroscience, 6, 1-7. https://doi.org/10.3389/fnint.2012.00099.

Johnson, B. P., Lum, J. A. G., Rinehart, N. J., \& Fielding, J. (2016). Ocular motor disturbances in autism spectrum disorders: Systematic review and comprehensive meta-analysis. Neurosceince and Biobehavioral Reviews, 69, 260-279. https:// doi.org/10.1016/j.neubiorev.2016.08.007.

Karatekin, C. (2007). Eye tracking studies of normative and atypical development. Developmental Review, 27, 283-348. https://doi.org/ 10.1016/j.dr.2007.06.006.

Kelly, D. J., Walker, R., \& Norbury, C. F. (2013). Deficits in volitional oculomotor control align with language status in autism spectrum disorders. Developmental Science, 16, 56-66. https://doi.org/10. 1111/j.1467-7687.2012.01188.x.

Kemner, C., van der Geest, J. N., Verbaten, M. N., \& van Engeland, H. (2004). In search of neurophysiological markers of pervasive developmental disorders: Smooth pursuit eye movements? Journal of Neural Transmission, 111, 1617-1626. https://doi.org/10.1007/ s00702-004-0164-5.

Kenet, T., Orekhova, E. V., Bharadwaj, H., Shetty, N. R., Israeli, E., Lee, A. K., Agam, Y., Elam, M., Joseph, R. M., Hämäläinen, M. S., \& Manoach, D. S. (2012). Disconnectivity of the cortical ocular motor control network in autism spectrum disorders. Neuroimage, 61, 1226-1234. https://doi.org/10.1016/j.neuroimage.2012.03.010

Kovarski, K., Siwiaszczyk, M., Malvy, J., Batty, M., \& Latinus, M. (2019). Faster eye movements in children with autism spectrum disorder. Autism Research, 12, 212-224. https://doi.org/10.1002/ aur.2054.

Landa, R. J., Gross, A. L., Stuart, E. A., \& Faherty, A. (2013). Developmental trajectories in children with and without autism spectrum disorders: The first 3 years. Child Development, 84, 429-442. https://doi.org/10.1111/j.1467-8624.2012.01870.x.

Lord, C., Rutter, M., DiLavore, P. C., Risi, S., Gotham, K., \& Bishop, S. (2012). Autism diagnostic observation schedule (2nd ed.). Torrance: Western Psychological Services.

Luna, B., Doll, S. K., Hegedus, S. J., Minshew, N. J., \& Sweeney, J. A. (2007). Maturation of executive function in autism. Biological Psychiatry, 61, 474-481. https://doi.org/10.1016/j.biopsych.2006. 02.030 .

Minshew, N. J., Luna, B., \& Sweeney, J. A. (1999). Oculomotor evidence for neocortical systems but not cerebellar dysfunction in autism. Neurology, 52, 917-922. https://doi.org/10.1212/wnl.52.5.917.

Mosconi, M. W., \& Sweeney, J. A. (2015). Sensorimotor dysfunctions as primary features of autism spectrum disorders. China Life Sciences, 58, 1016-1023. https://doi.org/10.1007/x11427-015-4894-4.
Mosconi, M., Kay, M., D'Cruz, A. M., Seidenfeld, A., Guter, S., Stanford, L. D., \& Sweeney, J. A. (2009). Impaired inhibitory control is associated with higher-order repetitive behaviors in autism spectrum disorders. Psychological Medicine, 39, 1559-1566. https://doi.org/ 10.1017/S0033291708004984.

Mosconi, M. W., Luna, B., Kay-Stacey, M., Nowinski, C. V., Rubin, L. H., Scudder, C., Minshew, N., \& Sweeney, J. A. (2013). Saccade adaptation abnormalities implicate dysfunction of cerebellardependent learning mechanisms in autism spectrum disorders (ASD). PLoS One, 8, e63709. https://doi.org/10.1371/journal. pone.0063709.

Mosconi, M. W., Wang, Z., Schmitt, L. M., \& Sweeney, J. A. (2015). The role of cerebellar circuitry alterations in the pathophysiology of autism spectrum disorders. Frontiers in Neuroscience, 9(296), 1-24. https://doi.org/10.3389/fnins.2015.00296.

Nebel, M. B., Eloyan, A., Nettles, C. A., Sweeney, K. L., Ament, K., Ward, R. E., Choe, A. S., Barber, A. D., Pekar, J. J., \& Mostofsky, S. H. (2016). Intrinsic visual-motor synchrony correlates with social deficits in autism. Biological Psychiatry, 79, 633-641. https://doi. org/10.1016/j.biopsych.2015.08.029.

Nowinski, C. V., Minshew, N. J., Luna, B., Takarae, Y., \& Sweeney, J. A. (2005). Oculomotor studies of cerebellar function in autism. Psychiatry Research, 137, 11-19. https://doi.org/10.1016/j. psychres.2005.07.005.

Rommelse, N. N. J., van der Stigchel, S., \& Sergeant, J. A. (2008). A review on eye movement studies in childhood and adolescent psychiatry. Brain and Cognition, 68, 391-414. https://doi.org/10.1016/ j.bandc.2008.08.025.

Rosenhall, U., Johansson, E., \& Gillberg, C. (1988). Oculomotor findings in autistic children. Journal of Laryngology and Otology, 102, 435439. https://doi.org/10.1017/s0022215100105286.

Rutter, M., Bailey, A., \& Lord, C. (2003). Social communication questionnaire. LA: Western Psychological Services.

Schmitt, L. M., Cook, E. H., Sweeney, J. A., \& Mosconi, M. W. (2014). Saccadic eye movement abnormalities in autism spectrum disorder indicate dysfunctions in cerebellum and brainstem. Molecular Autism, 5, 1-13. https://doi.org/10.1186/2040-2392-5-47.

Shirama, A., Kanai, C., Kato, N., \& Kashino, M. (2016). Ocular fixation abnormality in patients with autism spectrum disorder. Journal of Autism and Developmental Disorders, 46, 1613-1622. https://doi. org/10.1007/s10803-015-2688-y.

Sumner, E., Hutton, S. B., Kuhn, G., \& Hill, E. L. (2018). Oculomotor atypicalities in developmental coordination disorder. Developmental Science, 21, e12501. https://doi.org/10.1111/desc.12501.

Sutera, S., Pandey, J., Esser, E. L., Rosenthal, M. A., Wilson, L. B., Barton, M., Green, J., Hodgson, S., Robins, D. L., DumontMathieu, T., \& Fein, D. (2007). Predictors of optimal outcome in toddlers diagnosed with autism spectrum disorders. Journal of Autism and Developmental Disorders, 37, 98-107. https://doi.org/ 10.1007/s10803-006-0340-6.

Takarae, Y., Minshew, N. J., Luna, B., \& Sweeney, J. A. (2004a). Oculomotor abnormalities parallel cerebellar histopathology in autism. Journal of Neurology, 75, 1359-1361. https://doi.org/10.1136/ jnnp.2003.022491.

Takarae, Y., Minshew, N. J., Luna, B., Krisky, C. M., \& Sweeney, J. A. (2004b). Pursuit eye movement deficits in autism. Brain, 127, 2584 2594. https://doi.org/10.1093/brain/awh307.

Takarae, Y., Minshew, N. J., Luna, B., \& Sweeney, J. A. (2007). Atypical involvement of frontostriatal systems during sensorimotor control in autism. Psychiatry Research, 156, 117-127. https://doi.org/10. 1016/j.pscychresns.2007.03.008. 
Takarae, Y., Luna, B., Minshew, N. J., \& Sweeney, J. A. (2008). Patterns of visual sensory and sensorimotor abnormalities in autism vary in relation to history of early language delay. Journal of the International Neuropsychological Society, 14, 980-989. https:// doi.org/10.1017/S1355617708081277.

Travers, B. G., Bigler, E. D., Duffield, T. C., Prigge, M. D. B., Froehlich, A. L., Lange, N., Alexander, A. L., \& Lainhart, J. E. (2016). Longitudinal development of manual motor ability in autism spectrum disorder from childhood to mid-adulthood relates to adaptive daily living skills. Developmental Science, 20(4). https://doi.org/10. 1111/desc. 12401.

Trevarthen, C., \& Delafield-Butt, J. (2013). Autism as a developmental disorder in intentional movement and affective engagement. Frontiers in Integrative Neuroscience, 7, 49. https://doi.org/10. 3389/fnint.2013.00049.
Wechsler, D. (2003). Wechsler intelligence scales for children (4th ed.). San Antonio: The Psychological Corporation.

Whyatt, C., \& Craig, C. (2013). Sensory-motor problems in autism. Frontiers in Integrative Neuroscience, 7, 1-12. https://doi.org/10. 3389/fnint.2013.00051.

Wilkes, B. J., Carson, T. B., Patel, K. P., Lewis, M. H., \& White, K. D. (2015). Oculomotor performance in children with high-functioning autism spectrum disorders. Research in Developmental Disabilities, 38, 338-344. https://doi.org/10.1016/j.ridd.2014.12.022.

Publisher's Note Springer Nature remains neutral with regard to jurisdictional claims in published maps and institutional affiliations. 